\title{
Prediction of electrical contact endurance subject to micro-slip wear using friction energy dissipation approach
}

\author{
Xiangjun JIANG ${ }^{1,2,3,{ }^{*}}$, Fengqun PAN ${ }^{1}$, Guoqiang SHAO ${ }^{2}$, Jin HUANG ${ }^{1}$, Jun HONG $^{2}$, Aicheng ZHOU $^{4}$ \\ ${ }^{1}$ Key Laboratory of Electronic Equipment Structural Design, Xidian University, Xi'an 710071, China \\ ${ }^{2}$ State Key Laboratory for Manufacturing System, Xi'an Jiaotong University, Xi'an 710049, China \\ ${ }^{3}$ State Key Laboratory of Structural Analysis for Industrial Equipment, Dalian University of Technology, Dalian 116024, China \\ ${ }^{4}$ Guilin University of Aerospace Technology, Guilin 541004, China
}

Received: 12 December 2017 / Revised: 05 February 2018 / Accepted: 06 July 2018

(C) The author(s) 2018. This article is published with open access at Springerlink.com

\begin{abstract}
Fretting wear is a common cause of failure of an electrical contact (EC). In this study, we analyzed in detail the failure of EC induced especially by sliding using the representative electrical terminals. Furthermore, combining the friction energy dissipation theory, we proposed a prediction model to evaluate the electrical connector endurance (ECE) based on the contact stress and geometrical changes during the wear process obtained from a numerical model. The study helps establish that the friction energy dissipation theory is a powerful tool to analyze a contact failure due to wear. The proposed model proves to be effective in predicting the ECE for all considered cases such as micro-slip amplitude, contact force, overturning angle, superficial layer thickness, and friction/wear coefficients.
\end{abstract}

Keywords: electrical connector endurance; fretting wear; friction energy dissipation; finite element analysis

\section{Introduction}

An electrical contact (EC) is a critical aspect of the electrical connections in both high-power (e.g. automotive electronics) and low-power (e.g. telephone connections) applications. The performance of an EC is strongly influenced by a number of factors such as vibration, pressure, temperature, etc. For example, an intense vibration causes alternating microdisplacements at the surface of an EC inducing wear, corrosion, and fatigue that affects its electrical conductance adversely [1]; therefore, an increase in contact resistance is considered as an indication of the failure of an EC.

The environmental factors have a significant effect on the failure of the EC. Fretting frequency is an important controlling factor in a fretting corrosion test because corrosion is a function of time. Tristani et al. [2] tested the multi-contacts at $30-80 \mathrm{~Hz}$ for fretting corrosion to study the vibration thresholds.
Kim et al. [3] found the hydrocarbon contaminants increasing the contact resistance of EC drastically. Varenberg et al. [4] found that the system stiffness could affect the wear rate.

In recent years, several works focused on the experiments and simulations of the fretting wear in ECs. The reliability of EC in an electronic device was studied by Hirosaka et al. [5]. It was found that the fretting wear, the wear of the tin, and the deposition of the oxide increased the contact resistance resulting in failures. Weißenfels et al. [6] studied the mechanism of electrical contact for the coupled electrical-thermalmechanical conditions whereby the experimental data were used to verify the numerical simulation using a new formula. A microcontact model for the contact surface was developed by Sepehri et al. [7]. Two sets of approximate formulae to calculate the normal and tangential forces were deduced from this model. Rochdi et al. [8] studied the copper automotive

* Corresponding author: Xiangjun JIANG, E-mail: xjjiang@xidian.edu.cn 


\begin{tabular}{|c|c|c|c|}
\hline \\
\hline \multicolumn{4}{|r|}{ Wear amplification factor } \\
\hline \multicolumn{4}{|c|}{$\begin{array}{l}\text { Contact width }(\mathrm{mm}) \\
\text { Power function coefficients }\end{array}$} \\
\hline E & Elastic modulus (GPa) & $p_{\mathrm{i}, \mathrm{j}}$ & Contact stress for the $j^{\text {th }}$ wear increment \\
\hline$E_{\mathrm{d}}$ & Energy dissipation function $(\mathrm{J})$ & & at node $\mathrm{i}(\mathrm{Pa})$ \\
\hline$e$ & Coating thickness $(\mu \mathrm{m})$ & $p(x)$ & Contact stress $(\mathrm{Pa})$ \\
\hline$e_{\text {ref }}$ & Reference coating thickness $(\mu \mathrm{m})$ & $R$ & Contact radius of terminals $(\mathrm{mm})$ \\
\hline$F_{\mathrm{c}}$ & Hertz contact force $(\mathrm{N})$ & $\Delta R$ & ECR variation \\
\hline$H$ & Materials hardness $\left(\mathrm{N} / \mathrm{mm}^{2}\right)$ & $\Delta R c$ & Threshold of ECR variation \\
\hline \multirow{2}{*}{$\Delta h_{\mathrm{i}, \mathrm{j}}$} & \multirow{2}{*}{$\begin{array}{l}\text { Wear depth for the } j^{\text {th }} \text { wear increment, } \\
\text { node i on either contact surface }\end{array}$} & $S$ & Total slip displacement (mm) \\
\hline & & $\Delta s_{\mathrm{i}, \mathrm{j}}$ & Contact depth for the $\mathrm{j}^{\text {th }}$ wear increment \\
\hline$h_{\mathrm{p}}$ & Thickness of plug terminal (mm) & & at node $\mathrm{i}$ \\
\hline$h_{\mathrm{s}}$ & Thickness of socket terminal (mm) & $\mathrm{u}$ & Effect factors \\
\hline \multirow[t]{2}{*}{ I } & \multirow{2}{*}{$\begin{array}{l}\text { Moment of inertia of socket terminal } \\
\text { contact } \operatorname{arm}\left(\mathrm{mm}^{4}\right)\end{array}$} & $V$ & Total wear volume $\left(\mathrm{mm}^{3}\right)$ \\
\hline & & $\mathrm{x}, \mathrm{y}$ & Cartesian coordinates \\
\hline K & Thickness of plug terminal & $\alpha, \beta, \gamma$ & Power function coefficients \\
\hline \multirow[t]{2}{*}{$k^{\prime}$} & Non-dimentional wear coefficients of & $\delta$ & Fretting slip amplitude (mm) \\
\hline & Archard & $\delta^{*}$ & Slip amplitude of two contact surfaces \\
\hline$k$ & Archard wear coefficients $\left(\mathrm{Pa}^{-1}\right)$ & & $(\mathrm{mm})$ \\
\hline \multirow[t]{2}{*}{$l$} & \multirow{2}{*}{$\begin{array}{l}\text { Contact arm length of socket terminal } \\
(\mathrm{mm})\end{array}$} & $\theta$ & Contact arm angle of socket terminal $\left(^{\circ}\right)$ \\
\hline & & $\theta_{\mathrm{s}}$ & Overturning angle $\left(^{\circ}\right)$ \\
\hline$m$ & Power function coefficient & $\mu$ & Friction coefficient \\
\hline$N_{\mathrm{t}}$ & Total wear cycles (cycles) & $v$ & Poisson ratio \\
\hline & Durability life of EC (cycle) & $\phi_{\mathrm{f}}$ & \multirow{2}{*}{$\begin{array}{l}\text { Energy dissipation density function } \\
\left(\mathrm{J} / \mathrm{mm}^{2}\right)\end{array}$} \\
\hline & \multicolumn{2}{|l|}{ Rference durability life (cycle) } & \\
\hline
\end{tabular}

connectors with a 2-micron-thick tin layer. The fatigue properties of EC were corresponded with the number of plugs, the elastic deflection of the connecting piece, and the change of contact surface. The electrical contact resistance (ECR) endurance of Ag coating under fretting wear was predicted using the energy density approach proposed by [9-10].

A few studies have successfully simulated the fretting wear using the finite element method (FEM). Xie and Chen [11, 12] used ABAUQS (a software for FEM) to simulate the fretting wear of the needle-type tincoated socket connector based on the energy density approach to establish the relationship between the vibration threshold level and the contact interface dynamics. Yoshihiro et al. [13] studied the effect of surface interactions of iron samples on photoelectron emission (PE) as a function of temperature, where the surfaces were scratched in air, water, and organic environments. Myshkin et al. [14] analyzed the micro- morphology and roughness of the contact surface using the improved Archard model, and established that the failure mechanism of the EC was primarily caused by the environmental factors.

The FEM-based nodal movement technology enables effective simulation of the fretting wear through investigation of removal process in the contact surface. Ding et al. [15] adopted Archard theory to demonstrate the ability of FEM in simulating the fretting wear quantitatively. In their subsequent research, Ding et al. [16] simulated the effect of debris, accumulated during fretting wear, on contact-surface morphology. Eventually, the interaction between fretting wear and cyclic plasticity in Ti-6Al-4V, an alpha-beta titanium alloy, was investigated based on the Archard model [17].

In the present research work, we propose an approach to simulate the fretting wear of an electrical contact quantitatively, whereby we simulate a sliding wear by calculating the wear depth of each sub-step using 
a modified Archard's equation that was implemented into an FEM model. A prediction model for the electrical contact endurance life with a modified power formulation is introduced to characterize the evolution of electrical contact resistance (ECR) as a function of fretting cycle, based on the research by Fouvry et al. [18, 19]. The critical parameters such as wear volume, contact pressure during the wear process, and final contact area resulting from failure of EC are obtained. Using these parameters, we determined the coefficients of the power function for prediction of ECR endurance in a global scenario with different design variables.

\section{Finite element modeling}

\subsection{Finite element model of EC}

An EC is usually achieved by a plug-and-socket connection. The relative motion between the two terminals is inevitable causing the fretting wear characterized by a number of scratches and peeling-off on the contact surface. The structure and size of a typical plug-andsocket (Fig. 1) used in a vehicle is selected as the EC for this research.

The contour of EC (Fig. 1) can be considered to form a line contact; therefore, the three dimensional structure could be constructed as a two dimensional model in the analysis. Because the terminals bear the contact load on both sides with symmetrical characters in the contact states (contact stress and micro-slip), the FEM model can be simplified as one half of the two dimensional model shown in Fig. 2. The finite element mesh of the terminals is divided by using 8 -node quadrilateral elements, and the surface contact element (CONTA172) and the target cell (TARGE169) of frictional-contact type are used

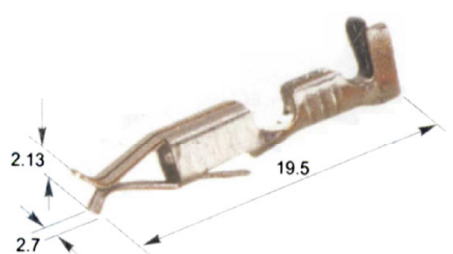

(a)

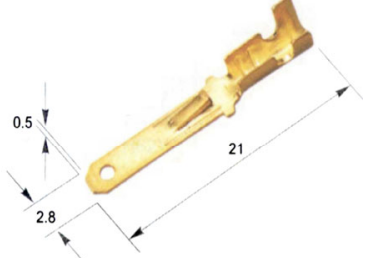

(b)
Fig. 1 Electric connecting piece (a) socket terminal; (b) plug terminal.

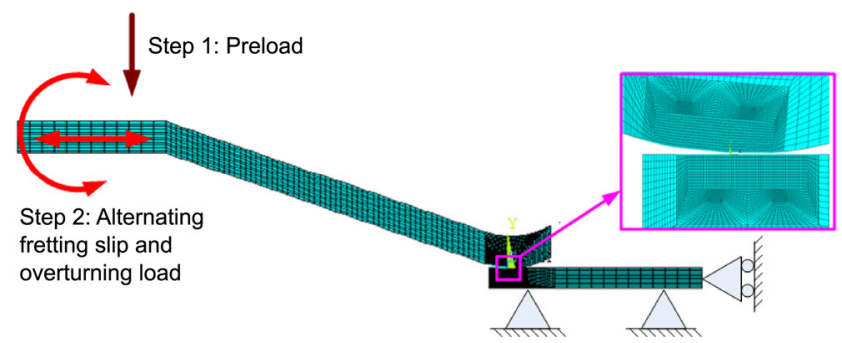

Fig. 2 Finite element modeling diagram.

to describe the contact interface between the plug and socket. The mesh grid of the FEM model at the contact region should be refined to capture the contact stress and micro-slip accurately. The mesh width is approximate $2.2 \mu \mathrm{m}$ at the contact region as per the refinement method in Ref. [15].

The accuracy of the contact stress calculation is a precondition for the validity of the wear analysis by FEM model, while the contact stress depends directly on the density at the contact surface. In order to verify the rationality of finite element mesh model, the accuracy is compared by using the Hertz contact theory.

Firstly, the contact arm of the socket terminal is considered as a rectangular cantilever beam, as shown in Fig. 3. The pre-tightening produces a contact force $F_{\text {c. }}$ The offset of the contact point is $h_{\mathrm{p}} / 2$ along the $Y$ direction in the coordinate axis, as shown in Fig. $3, h_{\mathrm{p}}$ is the thickness of the plug terminal. According to the deflection theory of beam, the following equation can be obtained,

$$
F_{\mathrm{c}}=\frac{3 E I h}{3 l^{3} \cos ^{2}(\theta)}
$$

where $l$ is contact arm length of socket terminal, $E$ is elastic modulus of terminal materials, $\theta$ is the contact

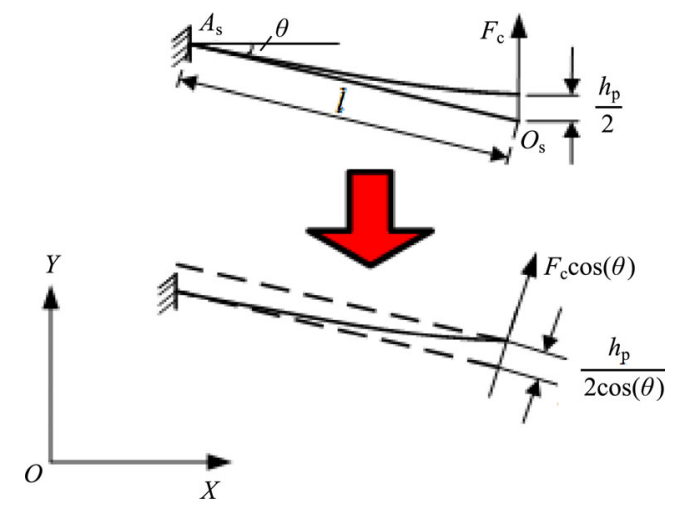

Fig. 3 Load analysis of contact arm of socket terminal. 
arm angle, moment of inertia $I=b h_{\mathrm{s}}^{3} / 12, b$ is the contact width of the socket and plug terminals, and $h_{\mathrm{s}}$ is the thickness of the socket terminal.

Therefore, according to the Hertz contact theory [20], the contact stress distribution can be written as,

$$
p(x)=\frac{3 F_{c}(1-x / a)^{2}}{\pi a}=\frac{3 E I h_{p}(1-x / a)^{2}}{\pi a l^{3} \cos ^{2}(\theta)}
$$

where the independent variable $x \in(-a, a), a$ is the contact width under contact force $F_{\mathrm{c}}$ and written as,

$$
a=\sqrt{\frac{4 F_{c} R}{\pi E^{*}}}=\sqrt{\frac{6 E I h_{p} R}{\pi E^{*} l^{3} \cos ^{2}(\theta)}}
$$

where $R$ is the geometry radius of socket terminal in contact region.

Combining Eq. (1), Eq. (2), and Eq. (3), the distribution of the contact pressure can be solved. A comparison between this solution and the finite element values, as shown in Fig. 4, confirms the rationality of the FEM model for the calculation of contact stress of the EC.

\subsection{Modeling for wear calculation}

The phenomenon model, such as Archard's wear model, has a strong inclusive and practical application [15] for wear simulation. The key parameters of Archard model are the contact pressure and relative sliding distance. Its expression form is as follows,

$$
a=\sqrt{\frac{4 F_{c} R}{\pi E^{*}}}=\sqrt{\frac{6 E I h_{p} R}{\pi E^{*} l^{3} \cos ^{2}(\theta)}}
$$

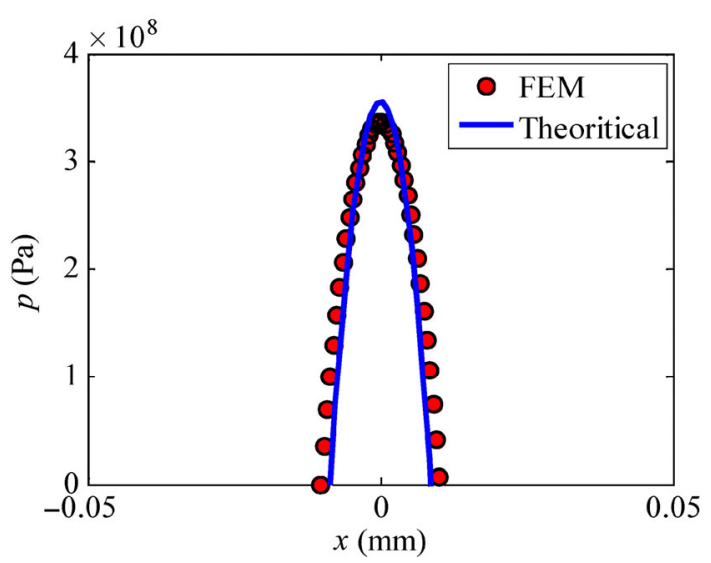

Fig. 4 Comparison between finite element analysis and calculation using Eq. (2).
In the formula, $V$ depicts the wear volume, $S$ is the sliding distance, $K$ means the non dimensional wear coefficient, $P$ denotes the normal force on contact surface, and $H$ is the hardness of the material.

Owing to the wear process, the profile of contact surface and the distribution of contact pressure would be changed continuously. In order to obtain an accurate contact pressure, the wear process should be divided into a number of wear sub-steps. Assuming that the sliding distance is $\mathrm{d} A$, the Archard model can be obtained from the $\mathrm{d} S$ within the unit contact area,

$$
\frac{\mathrm{d} V}{\mathrm{~d} S}=k \frac{P}{H}
$$

Dividing both sides by $\mathrm{d} A$,

$$
\frac{\mathrm{d} V}{\mathrm{~d} S \mathrm{~d} A}=k \frac{P}{H \mathrm{~d} A}
$$

In the formula, $P / \mathrm{d} A$ is a local contact pressure, which is a function of the position of the level contact points, denoted as $P(x) ; \mathrm{d} V / \mathrm{d} A$ is the local wear depth, denoted as $\mathrm{d} h ; K / H$ is considered as $k$, then the Eq.(6) can be rewritten as,

$$
\frac{\mathrm{d} h}{\mathrm{~d} S}=k p(x)
$$

When the wear process is discretized into a number of wear calculation steps, the cumulative amount of wear of each step is,

$$
h_{j+1}=h_{j}+k p_{j} \Delta s_{j}
$$

In the formula, $h_{\mathrm{j}}$ and $h_{\mathrm{j}+1}$ are the wear amount of the $\mathrm{j}$ and $\mathrm{j}+1$ moments, respectively, and $p_{\mathrm{j}}$ and $\Delta s_{\mathrm{j}}$ are the contact pressure and the sliding distance, respectively for the $j^{\text {th }}$ step. The wear rate and total accumulated wear can be calculated by the contact pressure $p$ and the sliding distance $s$ by Eqs. (6-8). Wear coefficient $k$ is associated with many factors such as the material properties, operating conditions, and so on.

The wear factor can be introduced into the wear simulation model to expedite the simulation that had been validated as an obvious method for reducing the simulation time [15-17],

$$
\Delta h_{i, j}=k \times 4 \Delta s_{i, j} \times \Delta N \times p_{i, j}
$$

where $\Delta N$ represents the wear amplification factor or 
the increment in number of wear cycles per step. For the $\mathrm{j}^{\text {th }}$ wear increment, at a given node $\mathrm{i}$ on either contact surface, the wear depth $\Delta h_{i, j}$, contact stress $p_{i, j}$, and slip magnitude $\Delta s_{i, j}$ are obtained from the finite element calculation. In order to expedite the wear process, the wear surface must be kept as smooth as possible simultaneously, the suitable value of the wear amplification factor $\Delta N$ should be obtained for both the stability of the simulation and the resulting computational time. In the course of simulation, the material removal and geometric shape updating caused by wear can be simulated by changing the position of the nodes and the shape of the surface mesh. The control strategy for boundary node mobility and mesh updating had been described in Ref. [21] in more details.

\subsection{Validation of the model}

In order to demonstrate the rationality of the FEM model for the wear process of EC, an experimental data of the cylinder-on-flat wear [15] is adopted to verify the validity of its simulation using ANSYS (a commercial finite element code) and the modified version of Archard's equation with the critical technologies to simulate both the fretting wear and the evolution of fretting variables with number of wear cycles. The critical technologies include the density grid design in the FEM model, the nodal movement technology, and the acceleration strategy for calculation.

The FEM model is shown in Fig. 5, where the radius of the cylinder is the same as the cylindrical specimen in the fretting tests [15]. Two-dimensional, four-node,

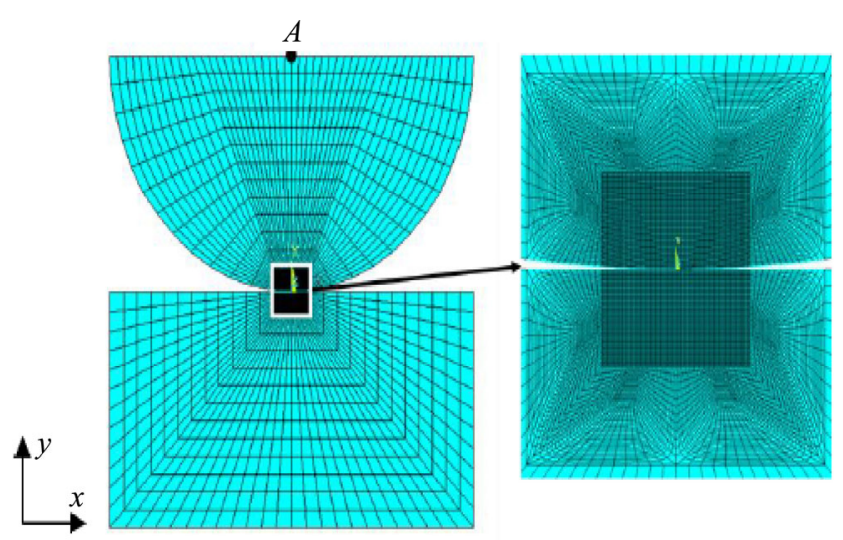

Fig. 5 Finite element mesh for cylinder-on-flat fretting wear model. plane strain (linear) elements are employed throughout. The mesh size in the contact area is very fine (approximately $20 \mu \mathrm{m}$ ) to capture the complicated variation of the stresses (surface and sub-surface) and relative slip. The basic Coulomb friction model with isotropic friction is employed. The elastic modulus, Poisson's ratio, and stable friction coefficient of both the cylinder and flat are taken as $200 \mathrm{GPa}, 0.3$, and 0.8, respectively. The wear coefficients for the case in this research are $3 \times 10^{-8} \mathrm{MPa}^{-1}$ and $2 \times 10^{-8} \mathrm{MPa}^{-1}$ for flat specimen and cylinder specimen, respectively.

The comparisons are firstly made with the wellknown analytical solutions of the Hertzian stress distributions to demonstrate the accuracy of the unworn model. The stress concentration would not be kept off as a concentrated force is applied at point A. That may lead to larger calculation deviation. Therefore, the multi-point couplings method is employed in $y$ direction for the node set with the same coordinate value as the point $\mathrm{A}$ in $y$ direction. The FEM model is observed to be in excellent agreement with the analytical solution for the distribution of contact pressure as shown in Fig. 6, thus indicating rational mesh refinement.

The cylinder-on-flat specimen is subjected to a fixed normal contact loading with cyclic tangential displacement in the simulation of fretting wear. The loading step for the finite element model is as follows: a normal load $185 \mathrm{~N}$ is applied to point $\mathrm{A}$ in the $\mathrm{y}$ direction, in one analysis step, and then a periodic $x$-displacement of an amplitude of $25 \mu \mathrm{m}$ is imposed at point A. The worn surface profiles of the flat

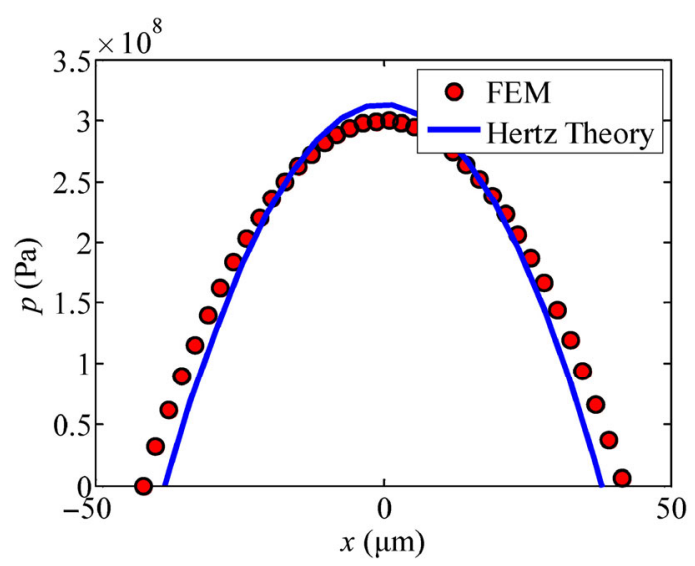

Fig. 6 Pressure distribution calculated by the finite element model and Hertz theory (Normal load: $185 \mathrm{~N}$ ). 
specimens by numerical prediction after 18,000 cycles have been compared with experimental results as presented in Fig. 7. The scar width and maximum wear depth for the predicted values are observed to be exactly matching with the experimental results.

Figure 8 shows the numerically predicted worn surface profiles with increasing fretting wear cycles, for the case of $185 \mathrm{~N}$ (normal load) and $25 \mu \mathrm{m}$ (displacement of amplitude). As the fretting wear proceeds, an increasing wear scar in the flat surface is observed, while the shape of the round surface is also modified. Both the contact surface profiles tend towards conforming with similar radii. The calculation results indicate that the present approach for fretting wear simulation, based on a modified Archard's equation, has excellent capability to predict the wear process of an electrical contact and its endurance life.

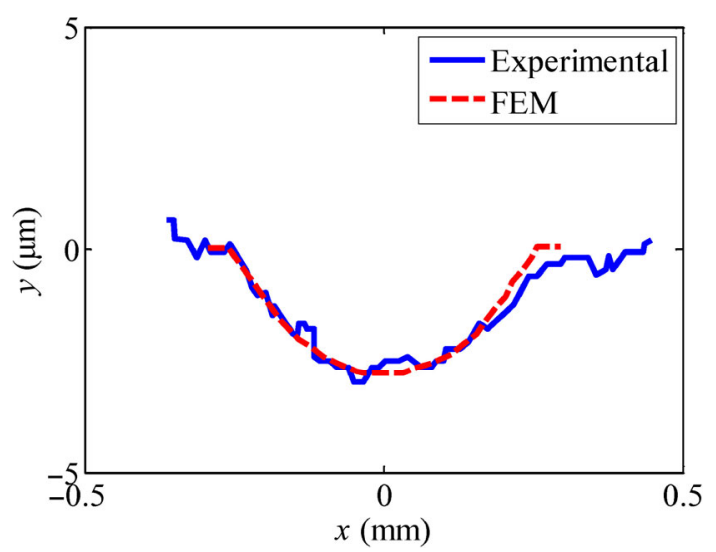

Fig. 7 Comparison of prediction and measured results for worn surface profiles of the flat specimen, after 18,000 wear cycles at $185 \mathrm{~N}$ and periodic displacement of amplitude of $25 \mu \mathrm{m}$.

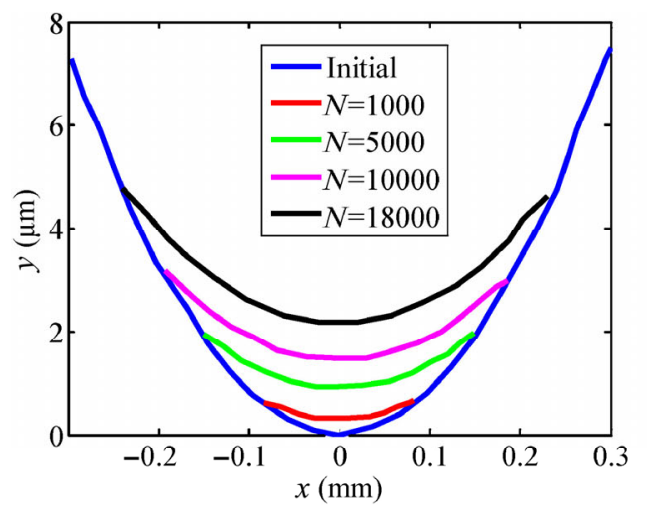

(a)

\section{Evolution of contact variables for electrical contact wear}

The studied interface consists of CuSn4-brass-alloy substrate on which a $2 \mu \mathrm{m}$ electrolytic-nickel interlayer is deposited to limit copper diffusion (Fig. 9). The superficial layer of the configuration is usual gold, silver, or tin to prevent oxidation of the EC, which is deposited on the structure using a dedicated electrolytic process. In this work, the wear coefficient $k=7.0 \times 10^{-16}$ and the friction coefficient $\mu=1.2$ that represent the values for a pure silver layer are used to simulate the wear process of the superficial layer of the EC, which is calculated as per Fouvry [18] through a combination of experiments and Archard's theory. The elastic modulus and Poisson's ratio of the superficial silver layer are taken as $120 \mathrm{GPa}$ and 0.34 , respectively, while those of the brass-alloy substrate and the electrolyticnickel interlayer are taken as $103 \mathrm{GPa}, 210 \mathrm{GPa}$, and 0.3 , respectively.

It can be found from the assembly processes of the EC that the plug and socket terminals should be housed in a casing. Because of the existence of a gap between the terminal and the casing, the plug and socket terminals are susceptible to move mutually along the horizontal and overturn directions as shown in Fig. 2, due to the deadweight of the connectors or the inertia of the wires at the terminals that are often subjected to vibrations. Considering the assembly conditions, the EC durability life is determined by several parameters including the materials of superficial layer, the thickness of superficial layer $e$, the contact

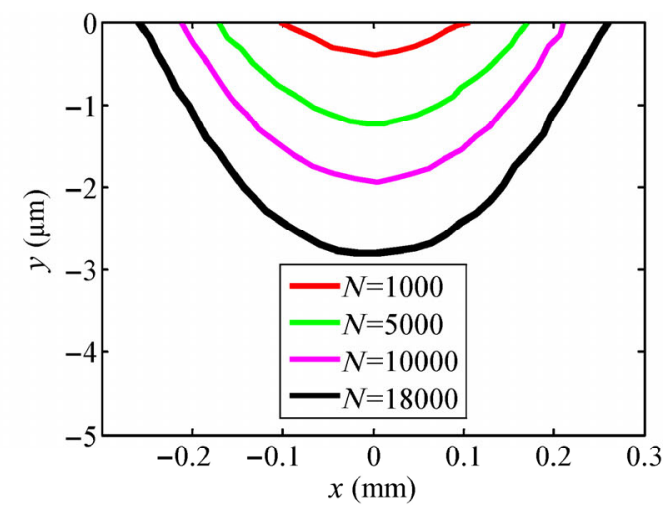

(b)

Fig. 8 Predicted wear profiles for: (a) cylindrical; and (b) flat specimens under $185 \mathrm{~N}$ normal load case, after different numbers of wear cycles $\mathrm{N}$. 


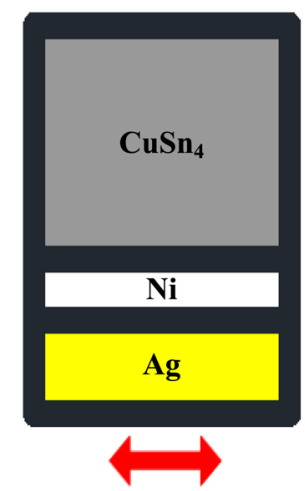

Fig. 9 Interface configuration of the homogeneous interface.

force $P$, and the vibration conditions in the form of alternating displacements $\delta$ in horizontal and overturn directions. It should be noted that in this research, the EC is considered as failed when the wear depth reaches the thickness of superficial layer $e$. The ECR endurance life is affected by a few critical wear parameters such as wear volume, contact pressure, and final contact area as the failure of EC depends on its materials, structure design, and the vibration environment; therefore, those parameters are firstly analyzed by the FEM calculation using the wear model as follows.

\subsection{Effect of fretting slip amplitude}

The electrical contact wear is initially caused by fretting slip under vibration conditions. It was found that the limitation of the slip amplitude for the EC is close to $4 \mu \mathrm{m}$ [18]. It means that the endurance life of the EC would be infinite owing to the partial slip wear within that slip amplitude; therefore, different fretting slip amplitudes $(\delta=25 \mu \mathrm{m}, 20 \mu \mathrm{m}, 15 \mu \mathrm{m}, 10 \mu \mathrm{m}$, and $5 \mu \mathrm{m}$ ) larger than $4 \mu \mathrm{m}$ are considered for the analyses of the wear morphology of electrical contact. The preload of electrical contact in this analysis is $h_{p} / 2=0.25 \mathrm{~mm}$, which produces a contact force $P=$ $13.1 \mathrm{~N}$. The thickness of superficial layer is $e=2 \mu \mathrm{m}$, and there is no overturning load.

Figures 10(a) and 10(b) show the worn surface profiles and contact pressure distributions under different fretting slip amplitudes for which the electrical contact is considered as failed. It is found that the distinction of wear scar width among these different cases is small. The wear form is typical of the whole contact slip wear according to the worn profile. The wear width increases with fretting slip amplitude. However, although the wear width is narrower for the smaller slip amplitude, the curvature of worn surface profile is more modest. Therefore, a reverse decreasing trend by comparison can be observed for the contact pressure distribution.

\subsection{Effect of preload}

The contact load is the most critical parameter for the wear process. For the EC, the preload force exerted during the assembly process (as the plug terminal is inserted into the socket terminal) is determined by the thickness of the plug terminal. According to the specimen design in Fig. 1, half of the thickness of plug terminal is $0.25 \mathrm{~mm}$ that would produce a preload force of $13.1 \mathrm{~N}$. Correspondingly, the half-the-thickness values of $0.15 \mathrm{~mm}$ and $0.05 \mathrm{~mm}$ would generate the preload forces of $7.9 \mathrm{~N}$ and $2.7 \mathrm{~N}$, respectively.

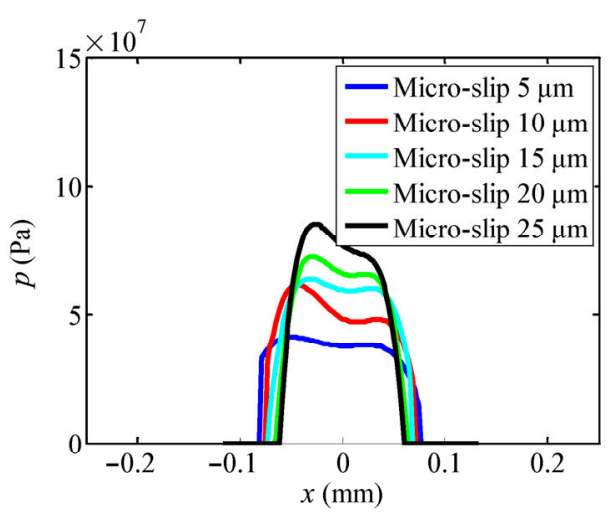

(a)

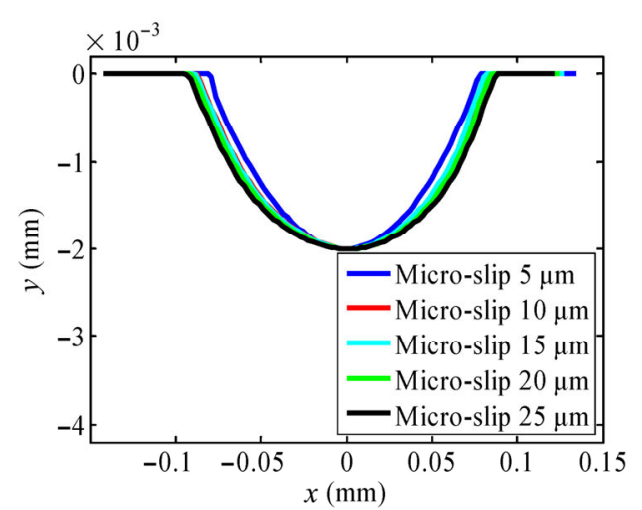

(b)

Fig. 10 Parameters evolution of wear under different fretting slip amplitude $\left(P=13.1 \mathrm{~N}, e=2 \mu \mathrm{m}, \theta_{s}=0^{\circ}\right)$ : (a) contact stress; $(\mathrm{b})$ worn surface profile. 
Figure 11 shows the contact pressure distributions under different preload cases as the EC reaches its durability life. It can be found that the contact peak pressure linearly decreases as the preload force is decreased, which corresponds to the initial contact peak pressure calculated by Eq. (2). The contact width shows a decrease form of power function formulation, which is consistent with Hertz contact form in Eq. (2) for the relationship between the contact width and the normal load.

\subsection{Effect of overturning angle}

The mutual rotating load was found to influence the wear behavior between parts of mechanical equipment [23-24]. The previous sections illustrated the existence of the mutual movement between the plug and socket

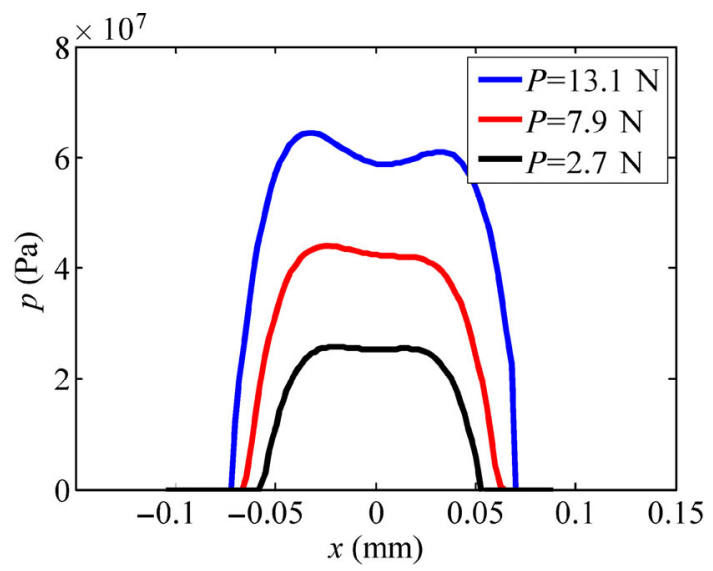

Fig. 11 Contact pressure evolution under different preload force $\left(\delta=25 \mu \mathrm{m}, e=2 \mu \mathrm{m}, \theta_{s}=0^{\circ}\right)$.

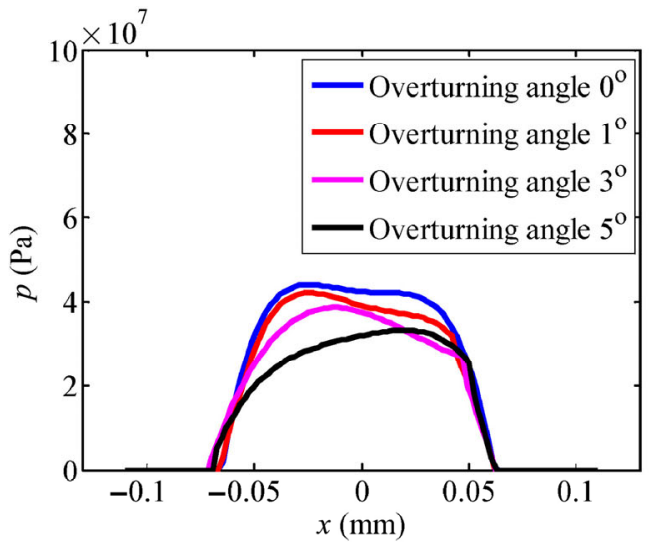

(a) terminals in overturning direction. It is difficult to restrain this movement due to the assembly clearance in the casing. In this section, the effects of different overturning angles on worn features of EC are discussed. Figure 12(a) shows the contact pressure distributions for different overturning angles. Owing to the modest worn profile, the contact pressure decreases with the increase of the overturning angle; however, as shown in Fig. 12(b), the worn profiles show asymmetry compared among one another. According to the FEM analyses, the contact surface shows larger contact pressure as the plus terminal rotates to the clockwise limitation compared with the case of the anti-clockwise limitation. This leads to the severer wear on the contact surface.

Figure 13 shows the effects of different slip amplitudes on worn features of EC for the case of overturning angle $\theta_{\mathrm{s}}=5^{\circ}$ and $P=7.9 \mathrm{~N}$. Similar to the Fig. 9, the lower slip amplitude leads to the smaller wear width and lower electrical contact pressure due to the more modest radius of curvature of the worn surface. Owing to the effect of overturning, the contact pressure relatively decreases with $30 \%$ amplitude approximately. This depicts that the overturning affects the worn features of the EC obviously. The identical conclusion can be obtained in the case of different preloads with a specific overturning as shown in Fig. 14; the overturning affects the surface wear adversely leading to a higher decrease of the contact pressure compared to the case of no overturning.

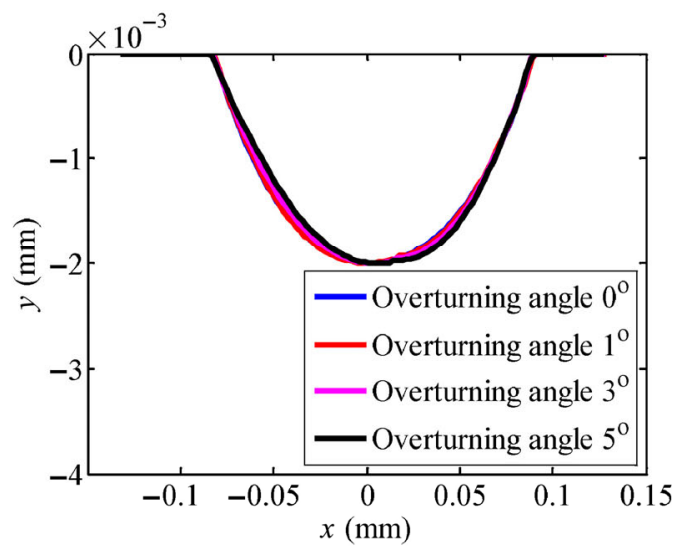

(b)

Fig. 12 Parameters evolution of wear under different overturning angles with $P=7.9 \mathrm{~N}, \delta=15 \mu \mathrm{m}$ : (a) contact pressure; (b) worn surface profiles. 


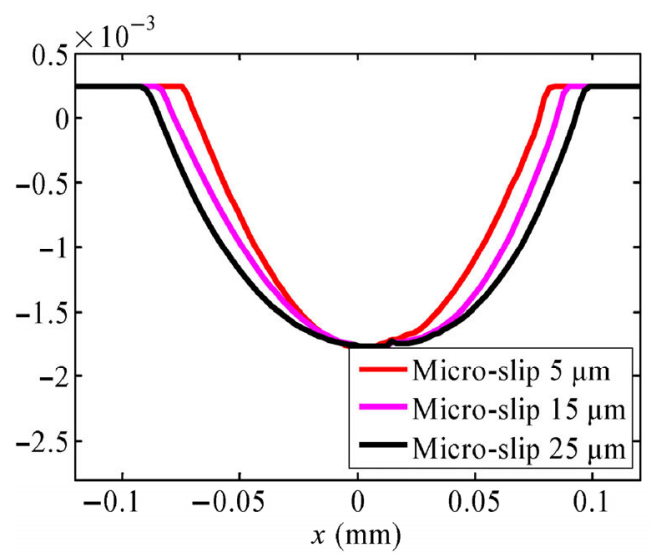

(a)

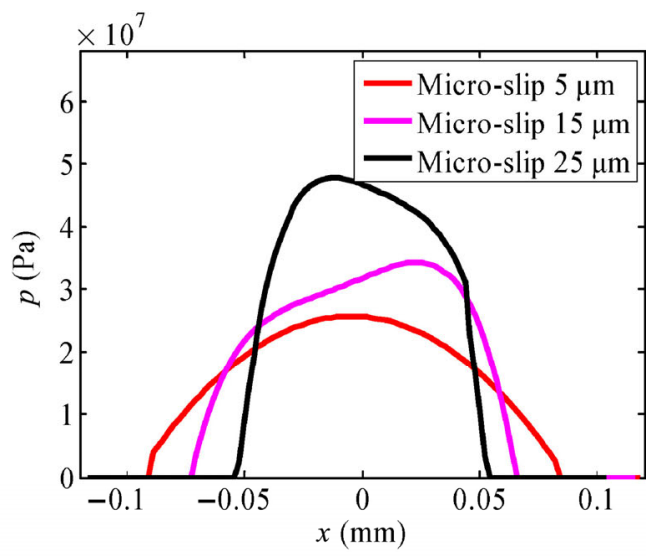

(b)

Fig. 13 Parameters evolution of wear under different fretting slip amplitudes with $P=7.9 \mathrm{~N}$ and $\theta_{\mathrm{s}}=5^{\circ}$ : (a) contact stress; (b) worn surface profile.

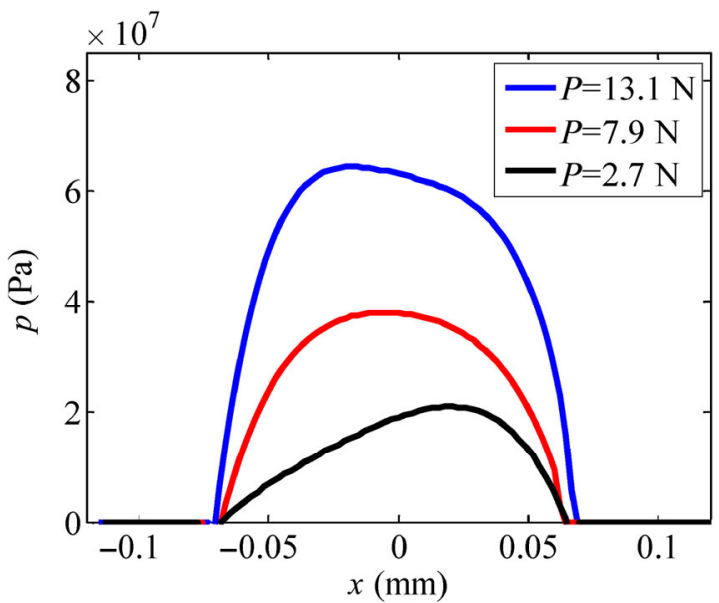

Fig. 14 Contact pressure under different preload for a specific overturning angle $\left(\delta=15 \mu \mathrm{m}, \theta_{\mathrm{s}}=3^{\circ}\right)$.

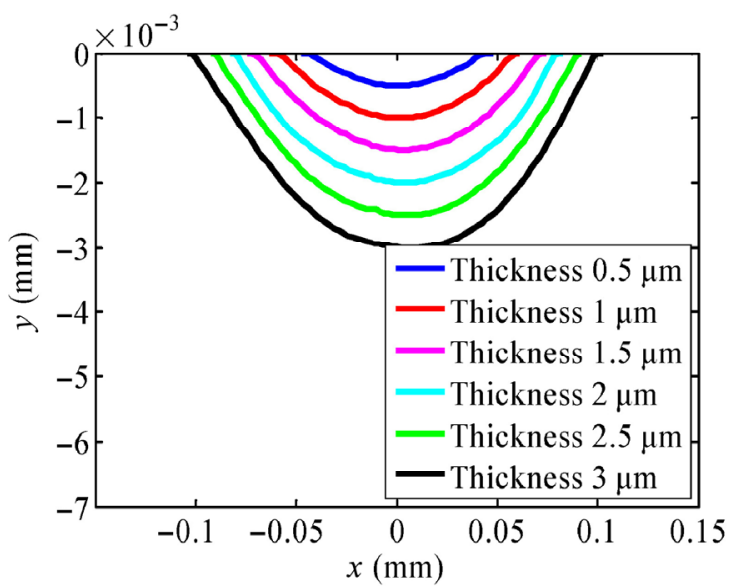

\subsection{Effect of superficial layer thickness}

The superficial layer usually consists of gold, silver or tin, which is the excellent antioxidant material. Once this layer is close to be worn, the electrical contact tends to fail. In this section, the effect of the thickness of the superficial layer on the contact features of the EC is discussed. Figure 15(a) shows the worn profiles for different thicknesses of superficial layer. The worn width increases with worn depth. Due to the contact arm of socket terminal, the contact interface can afford additional bending moment. This moment creates a little asymmetry for the geometric shape of the worn surface, which leads to an obvious asymmetry distribution of the contact pressure, as shown in Fig. 15(b).

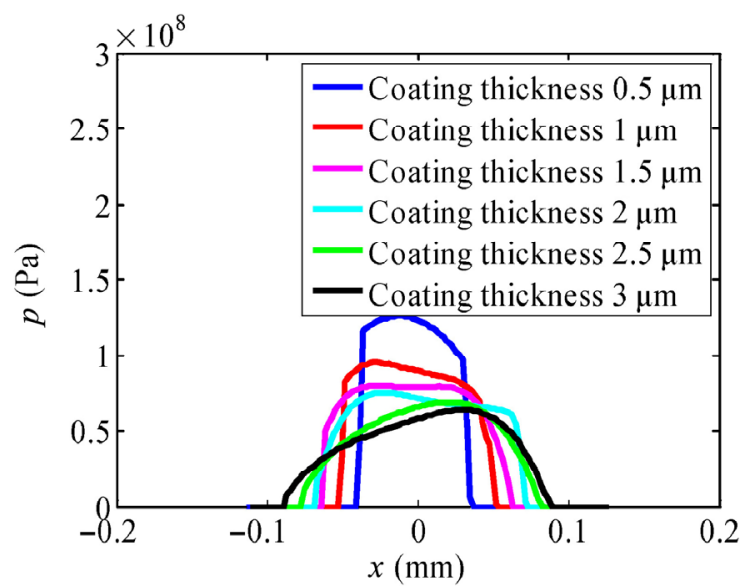

Fig. 15 Parameters evolution of wear under different thicknesses of superficial layer with $P=13.1 \mathrm{~N}, \delta=15 \mu \mathrm{m}, \theta_{s}=0^{\circ}$ : (a) worn surface profiles; (b) contact pressure. 


\subsection{Effects of friction and wear coefficients}

The friction and wear coefficients are critical parameters of the wear process. Figure 16(a) shows that the contact pressure decreases with the increase of the friction coefficient. According to the friction energy dissipation theory [22],

$$
E_{d}=4 \mu \delta P
$$

which indicates that larger the friction coefficient, higher the friction energy dissipation leading to severer wear on the contact surface. $E_{\mathrm{d}}$ is the friction energy dissipation function, $\mu$ is friction coefficient, $P$ depicts the contact force, and $\delta$ is the mutual movement displacement of the contact surfaces. Figure 16(b) shows that higher the wear coefficient, severer the wear thereby reducing the contact pressure considerably. It is also observed that both the friction and wear coefficients have little influence on the width of the contact region.

\section{Durability life prediction of EC}

\subsection{Modeling of the durability life prediction}

In the previous sections, we analyzed the effects of fretting slip amplitude, preload force, superficial layer thickness, and overturning angle on the wear characteristics of the EC, with the aim to obtain necessary input parameters for the prediction model of durability life of EC.

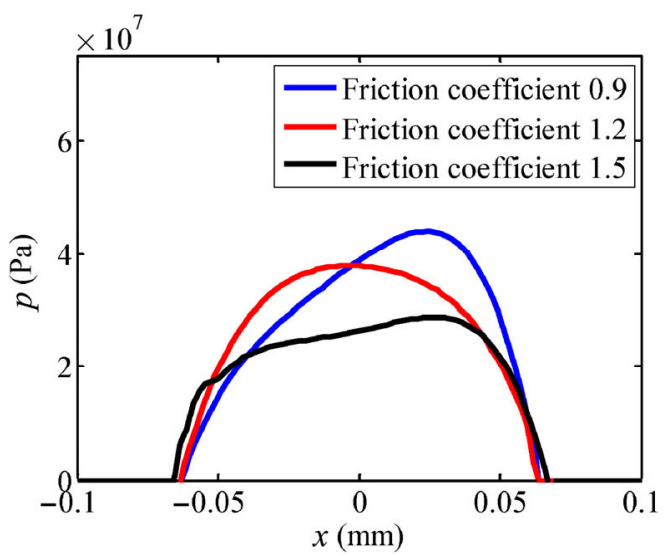

(a) Effect of friction coefficients
In this work, the EC is considered to be at the end of its durability life as the wear depth equals the superficial layer thickness. It implies that the EC loses its effectiveness once the superficial layer is worn out completely; however, the failure of EC should be confirmed from the electrical contact resistance. In Refs. [9, 18-19], the EC was considered as failed when the ECR variation reached a threshold value of $\Delta R>$ $\Delta R c=0.004 \Omega$ that corresponds to the ECE and below which no current micro-cuts were usually observed. However, the researches in Refs. $[9,18]$ found that the friction energy dissipation $\left(E_{\mathrm{d}}\right)$ or the wear depth in contact interface is correlated with the threshold $\Delta R \mathrm{c}$, where $E_{\mathrm{d}}$ is the mean friction energy dissipated per fretting cycle. It means that the ECR increases rapidly as the wear depth approaches the superficial layer thickness $e$. In other words, as the friction energy dissipation or the wear depth reaches a specific value, the EC can be considered as failed. In this research, the superficial layer thickness $e$ is defined as the effective wear depth; accordingly, the EC can be considered as failed as the wear depth reaches this critical value $e$ under transverse cyclic loadings. Therefore, the prediction model of the durability life of EC could be constructed using the function of the friction energy dissipation.

The analysis in Ref. [9] allowed the effective prediction of ECE life in a given fretting contact configuration, considering the fretting sliding amplitude, preload, and superficial layer thickness. The corresponding model was expressed as follows,

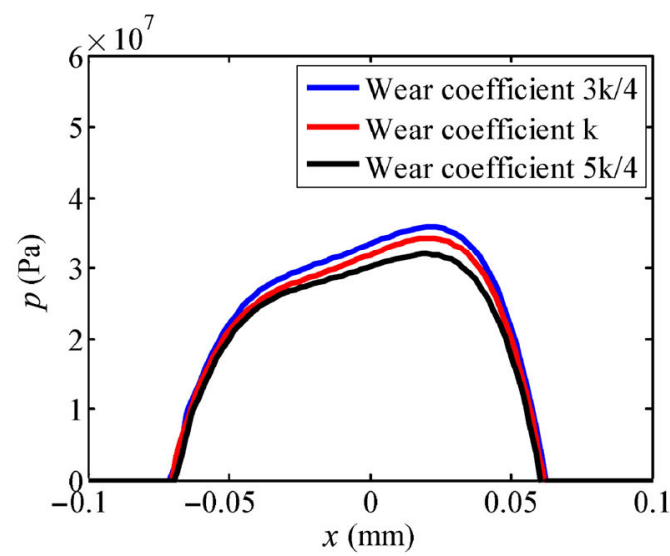

(b) Effect of wear coefficients

Fig. 16 Contact pressure for different friction and wear coefficients: (a) $P=7.9 \mathrm{~N}, \delta=15 \mu \mathrm{m}, \theta_{s}=3^{\circ}$; (b) $P=7.9 \mathrm{~N}, \delta=15 \mu \mathrm{m}, \theta_{s}=5^{\circ}$. 


$$
\begin{aligned}
N c & =N c_{0}\left(\frac{E_{\mathrm{d}}}{W c_{\mathrm{f}}}\right)^{\alpha}\left(\frac{e}{e_{\text {ref }}}\right)^{\beta} \\
& =N c_{0}\left(\frac{4 \mu \delta}{W c_{0}}\right)^{\alpha} P^{\alpha(1-m)}\left(\frac{e}{e_{\text {ref }}}\right)^{\beta}
\end{aligned}
$$

where the means $\delta, P$, and $e$ are fretting sliding amplitude, preload, and superficial layer thickness, respectively. $N c_{0}, m, \alpha, \beta$, and $W c_{0}$ are fixed parameters characterizing the materials. $\mu$ is the friction coefficient. $e_{\text {ref }}$ is the reference thickness of superficial layer. $W c_{0}$ is obtained from a power law approximation to describe the final contact width extension,

$$
W c_{\mathrm{f}}=W c_{0} P^{m}
$$

where $W c_{\mathrm{f}}$ indicates the wear width when the EC is on the verge of failure.

These coefficients can be obtained by experiments or finite element simulation. Using the procedures described in Section 3, these coefficients can be calculated as $N c_{0}=8.6 \times 10^{6}, \alpha=-1.01, W c_{0}=9.2 \times 10^{-5}$, $m=0.17, \beta=1.3$, and $e_{\text {ref }}=2 \mu \mathrm{m}$; however, this model proposed in [9] had not considered the effects of the relative overturning movement of contact bodies and the wear coefficient. In this work, an improved model is developed to estimate the influence of these two factors.

The friction energy dissipation can be considered as the most critical parameter to determine the ECE life. The overturning load, which can also be considered as a relative displacement load, can be written as $\delta_{a}=R \theta_{s}$. Therefore, the friction energy dissipation function can be rewritten as,

$$
E_{d}=4 \mu\left(\delta+\delta_{a}\right) P
$$

Furthermore, the wear volume is directly related to friction energy dissipation of the wear process. As the wear volume increases, the friction energy dissipation also increases. Coupling Eq. (13), the friction energy dissipation function is expressed as,

$$
E_{d}=4\left(k / k_{\text {ref }}\right) \mu \delta P=4 k^{\prime} \mu \delta P
$$

where $k^{\prime}=k / k_{\text {ref }}$ is an equivalent dimensionless wear coefficient. The reference wear coefficient $k_{\text {ref }}=7.0 \times 10^{-16}$ is the same value used for silver in Section 3.
Therefore, the prediction model can be updated as,

$$
N c=N c_{0}\left(\frac{4 k^{\prime} \mu(\delta+\delta)}{W c_{0}}\right)^{\alpha} P^{\alpha(1-m)}\left(\frac{e}{e_{\text {ref }}}\right)^{\beta}
$$

Therefore, the improved model could consider the fretting sliding amplitude, preload, superficial layer thickness, friction coefficient, wear coefficient, and overturning amplitude.

\subsection{Analyses of durability life}

In order to compare with the calculated results by predicted model, the finite element calculation results are presented by the fitting curve of power law. The fitting function is written as,

$$
N c^{\prime}=N c_{0}^{\prime} u^{\gamma}
$$

where $N c^{\prime}$ is the fitting endurance life by the finite element calculation results, $N c_{0}^{\prime}$ and $\gamma$ are fixed parameters. $u$ presents the effect factors such as the fretting slip amplitude $\delta$, the preload force $P$, the superficial layer thickness $e$, the friction coefficient $\mu$, the wear coefficient $k$, and the overturning angle $\theta_{s}$.

Figure 17 plots the calculated ECE of both FEM and proposed model. It can be found that the power law function is appropriate to express the ECE obtained from FEM as a function of the respective effect factors. It is evident that ECE decreases with increase in these effect factors except for the superficial layer thickness. The comparison with FEM shows that the proposed model has the capability to predict the ECE well by considering the effects of the overturning angle $\theta_{s}$ and the wear coefficient $k$. However, a small difference is observed in every case is explained by the computation process for the friction energy dissipation function. Equation (14) shows that the friction energy dissipation is assumed to be associated with the fretting slip amplitude $\delta$. In this research, the fretting slip amplitude $\delta$ is the external displacement load. However, the friction energy dissipation is actually produced by the relative sliding amplitude $\delta^{*}$ between the two contact surfaces. Owing to the existence of the local stick in the contact surfaces during the wear cycles, the relationship $\delta^{*}<\delta$ can be derived leading to a smaller prediction results of ECE. However, the difference between $\delta^{*}$ and $\delta$ is neglected in this research due 

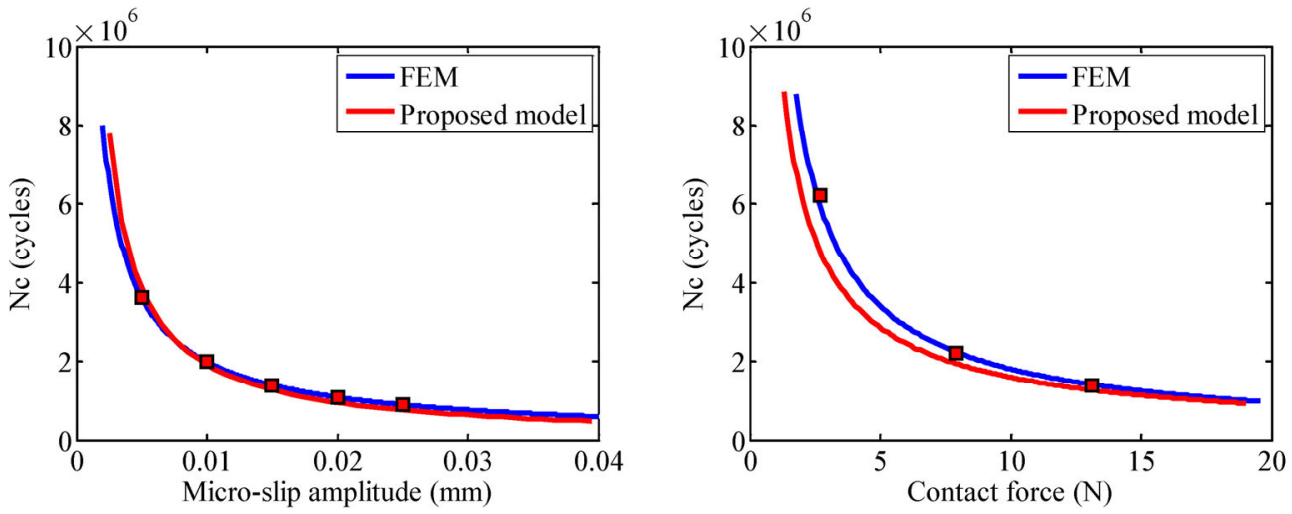

(a)

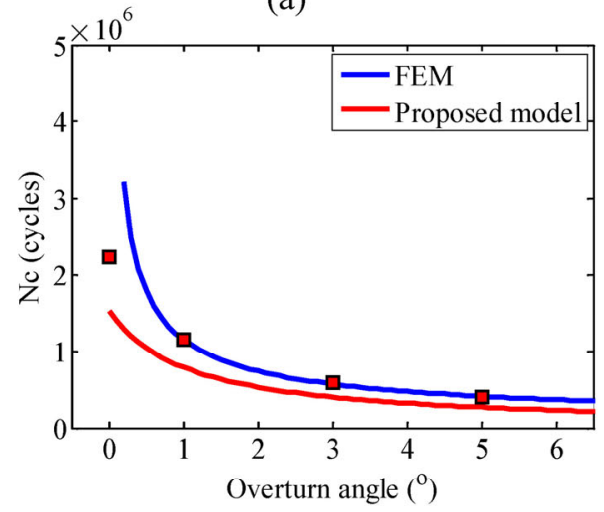

(b)

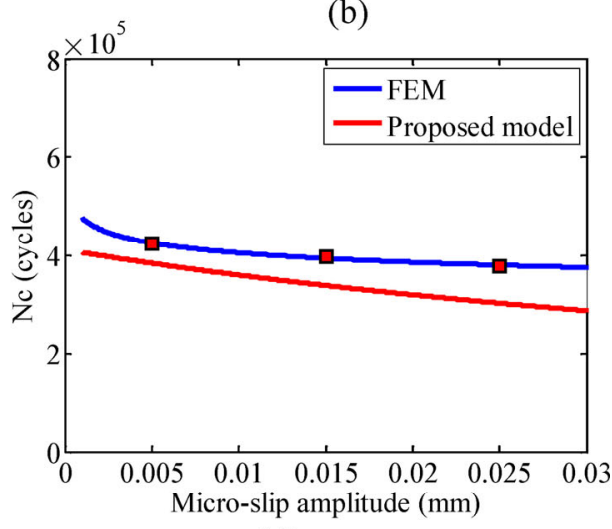

(c)

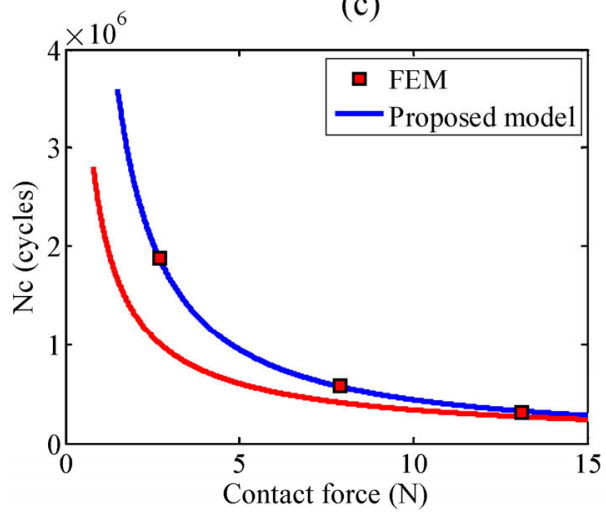

(d)

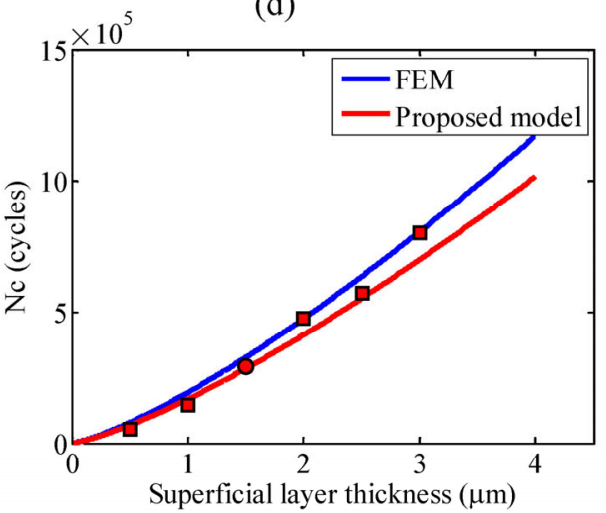

(e)

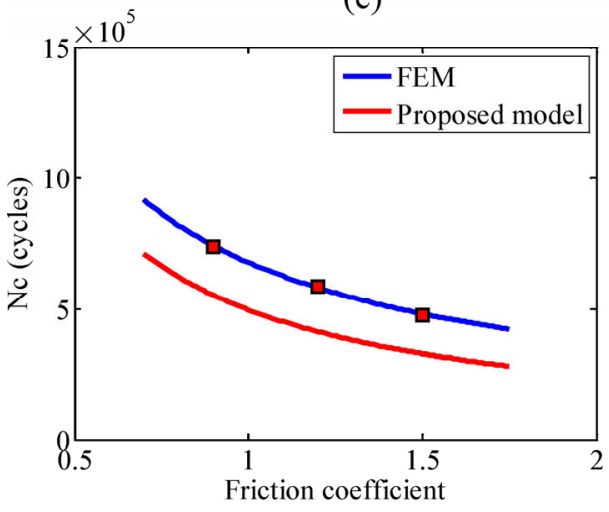

(g)

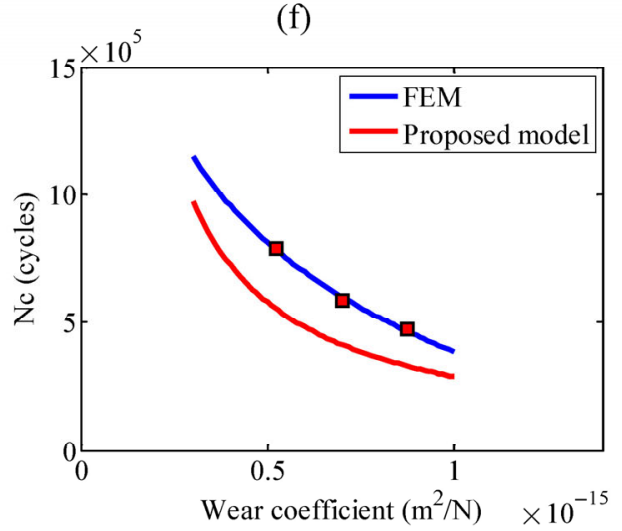

(h)

Fig. 17 ECE life prediction versus finite element analysis results for different effect factors: (a) micro-slip amplitude; (b) contact force; (c) overturning angle; (d) micro-slip amplitude with $\theta_{s}=5^{\circ}$; (e) contact force with $\theta_{s}=3^{\circ}$; (f) superficial layer thickness; (g) friction coefficient with $\theta_{s}=3^{\circ}$; (h) wear coefficient with $\theta_{s}=5^{\circ}$. 
to the complex mechanism of the relative sliding amplitude $\delta^{*}$ and the small relative influence on the calculated results of ECE.

\section{Conclusions}

In this study, we analyzed the failure of electrical contact induced by fretting using a sliding-based representative electrical connector (plug-and-socket). In addition, we proposed a prediction model to evaluate the ECE. The findings of the study are summarized as below.

1) The friction energy dissipation theory is a powerful tool to analyze a contact failure due to wear. It can be concluded from the finite element analyses that higher friction energy dissipation, lower the contact pressure and more moderate the geometry of electrical contact at the time of failure. For example, among the different fretting slip amplitudes investigated in the range of $5-25 \mu \mathrm{m}$, a micro-slip of $5 \mu \mathrm{m}$ with a very large number of loading cycles would cause the most intense energy dissipation leading to the lowest contact pressure after the failure of electrical contact. The larger friction/wear coefficients and overturning angle would cause larger energy dissipation for electrical contact wear. The corresponding lower contact pressure could also be observed from the finite element analyses.

2) The proposed model shows effectiveness in predicting the ECE for all considered cases. The assumption of the transformation of overturning angle to the slip displacement proves to be reasonable. The equivalent dimensionless wear coefficient incorporated in the friction energy dissipation function with proportional relationship is also found to be rational. Because a small local stick on the electrical contact surface during the wear cycles was neglected, the proposed model is observed to underestimate the ECE marginally compared to the FEM results. This shows that there is immense scope to investigate the effect of the local stick on ECE in detail. Similarly, there is also scope for future work to verify the theoretical results of the proposed model by suitable experiments.

\section{Acknowledgments}

We would like to thank for the financial support of this work by the National Natural Science Foundation of China (NSFC) under Grant Numbers 51775406 and 51405371, Open Research Fund of State Key Laboratory of Structural Analysis for Industrial Equipment (Grant No. GZ1612), 111 Project B14042, the Fundamental Research Funds for the Central Universities (Grant No. JB180412), Natural Science Foundation of Shanxi Province of China (Grant No. 2017JM5035), and Natural Science Foundation of Guangxi Province of China (Grant No. 2016GXNSFBA380230).

Open Access: The articles published in this journal are distributed under the terms of the Creative Commons Attribution 4.0 International License (http://creativecommons.org/licenses/by/4.0/), which permits unrestricted use, distribution, and reproduction in any medium, provided you give appropriate credit to the original author(s) and the source, provide a link to the Creative Commons license, and indicate if changes were made.

\section{References}

[1] Antler M. Tribology of electronic connectors: Contact sliding wear, fretting, and lubrication. In: Electrical Contacts: Principles and Applications. Slade P G, Ed. New York: Marcel Dekker Inc., 1999: 309.

[2] Tristani L, Zindine E M, Boyer L, Klimek G. Mechanical modeling of fretting cycles in electrical contacts. Wear 249(1-2): 12-19 (2001)

[3] Kim Y T, Sung I H, Kim J S, Kim D E. Effects of contact conditions on the connector electrical resistance of direct current circuits. Int Commun Heat Mass 5(3): 5-10 (2004)

[4] Varenberg M, Etsion I, Halperin G. Slip index: a new unified approach to fretting. Tribol Lett 17(3): 569-573 (2004)

[5] Ikeda H, Ito T, Sawada S, Hattori Y, Saitoh Y, Tamai T, Iida K. Influence of fretting wear on lifetime of tin plated connectors. IEICE Trans Electron E92.C(9): 1215-1222 (2009)

[6] Weißenfels C, Wriggers P. Numerical modeling of electrical contacts. Comput Mech 46(2): 301-314 (2010)

[7] Sepehri A, Farhang K. On elastic interaction of nominally flat rough surfaces. J Tribol 130(1): 011014 (2008)

[8] El Abdi R, Benjemaa N, Beloufa M. Numerical and experimental studies of automotive connector behavior. In: Proceedings of the 18th IASTED International Conference on Modelling and Simulation, Montreal, Quebec, Canada, 2007: 209-214. 
[9] Laporte J, Perrinet O, Fouvry S. Prediction of the electrical contact resistance endurance of silver-plated coatings subject to fretting wear, using a friction energy density approach. Wear 330-331: 170-181 (2015)

[10] Fouvry S, Liskiewicz T, Kapsa P, Hannel S, Sauger E. An energy description of wear mechanisms and its applications to oscillating sliding contacts. Wear 255(1-6): 287-298 (2003)

[11] Xie F, Flowers G T, Chen C, Bozack M, Suhling J, Rickett B I, Malucci R D, Manlapaz C. Analysis and prediction of vibration-induced fretting motion in a blade/receptacle connector pair. In Proceedings of the 53rd IEEE Holm Conference on Electrical Contacts, Pittsburgh, PA, USA, 2007: 222-228.

[12] Chen C, Flowers G T, Bozack M, Suhling J. Modeling and analysis of a connector system for the prediction of vibrationinduced fretting degradation. In Proceedings of the 55th IEEE Holm Conference on Electrical Contacts, Vancouver, British Columbia, Canada, 2009: 129-135.

[13] Momose Y, Suzuki D, Tsuruya K, Sakurai T, Nakayama K. Transfer of electrons on scratched iron surfaces: Photoelectron emission and X-ray photoelectron spectroscopy studies. Friction 6(1): 98-115 (2018)

[14] Myshkin N K, Petrokovets M I, Chizhik S A. Simulation of real contact in tribology. Tribol Int 31(1-3): 79-86 (1998)

[15] Ding J, Leen S B, McColl I R. The effect of slip regime on fretting wear-induced stress evolution. Int J Fatigue 26(5): 521-531 (2004)

[16] Ding J, McColl I R, Leen S B. The application of fretting wear modelling to a spline coupling. Wear 262(9-10):

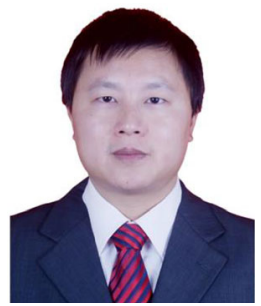

Xiangjun JIANG. He received his M.S. and Ph.D degrees in mechanical engineering from Xi'an Jiaotong University, China, in 2007 and 2013 respectively. He joined the Key

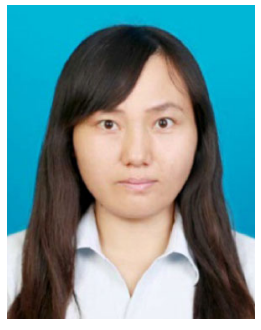

Fengqun PAN. She received her master's degree in chemical and mechanical engineering in 2012 from Shaanxi University of Science and Technology, Xi'an, China. After then,
1205-1216 (2007)

[17] McColl I R, Ding J, Leen S B. Finite element simulation and experimental validation of fretting wear. Wear 256(11-12): 1114-1127 (2004)

[18] Fouvry S, Jedrzejczyk P, Chalandon P. Introduction of an exponential formulation to quantify the electrical endurance of micro-contacts enduring fretting wear: Application to Sn, Ag and Au coatings. Wear 271(9-10): 1524-1534 (2011).

[19] Fouvry S, Jedrzejczyk P, Perrinet O, Alquier O, Chalandon P. Introduction of a "modified Archard wear law" to predict the electrical contact endurance of thin plated silver coatings subjected to fretting wear. In: Proceedings of the 58th IEEE Holm Conference on Electrical Contacts, Portland, OR, USA, 2012: 191-203.

[20] Johnson K L. Contact Mechanics. Cambridge (UK): Cambridge University Press, 1985.

[21] Schmitz T L, Action J E, Burris D L, Ziegert J C, Sawyer W G. Wear-rate uncertainty analysis. J Tribol 126(4): 802808 (2004)

[22] Fouvry S, Kapsa P, Vincent L. Quantification of fretting damage. Wear 200(1-2): 186-205 (1996)

[23] Wang X R, Wang D G, Zhang D K, Ge S R, Araújo J A. Effect of torsion angle on tension-torsion multiaxial fretting fatigue behaviors of steel wires. Int J Fatigue 106: 159-164 (2018)

[24] Wang D G, Li X W, Wang X R, Zhang D K, Wang D A. Dynamic wear evolution and crack propagation behaviors of steel wires during fretting-fatigue. Tribol Int 101: 348-355 (2016)

Laboratory of Electronic Equipment Structural Design at Xidian University from 2015. He is a lecturer of the laboratory. His research areas cover the tribology of electrical connector and wear simulation.

she was a Ph.D student of mechatronics engineering in the Key Laboratory of Electronic Equipment Structural Design at Xidian University. Her research interest is wear analysis by finite element method. 